\title{
DIFFERENTIATING ORLICZ SPACES WITH RARE BASES OF RECTANGLES
}

\author{
Emma D'Aniello, Laurent Moonens and Joseph M. Rosenblatt \\ Università degli Studi della Campania "Luigi Vanvitelli", Dipartimento di Matematica e Fisica \\ Viale Lincoln n. 5, 81100 Caserta, Italia; emma.daniello@unicampania.it \\ Université Paris-Sud, CNRS UMR8628, Université Paris-Saclay \\ Laboratoire de Mathématiques d'Orsay, Bâtiment 307 \\ F-91405 Orsay Cedex, France; laurent.moonens@math.u-psud.fr \\ University of Illinois at Urbana-Champaign, Department of Mathematics \\ 1409 W. Green Street, Urbana, IL 61801-2975, U.S.A.; rosnbltt@illinois.edu
}

\begin{abstract}
In the current paper, we study how the speed of convergence of a sequence of angles decreasing to zero influences the possibility of constructing a rare differentiation basis of rectangles in the plane, one side of which makes with the horizontal axis an angle belonging to the given sequence, that differentiates precisely a fixed Orlicz space. We also make a simple observation showing that the maximal operator associated to rectangles oriented in a fixed sequence of directions, is either bounded on all $L^{p}$ spaces for $1<p \leqslant \infty$, or fails to be bounded on any of them (adding the case $p=\infty$ to a dichotomy obtained previously by Bateman).
\end{abstract}

In the sequel we always call interval in $\mathbf{R}^{2}$ a set of the form $Q=[a, b] \times[c, d]$ where $a<b$ and $c<d$ are real numbers; a rectangle, on the other hand, will be any set obtained from an interval by some isometry of the plane.

There is a long history of research around the maximal operator $M_{\boldsymbol{\theta}}$ associated to a set $\boldsymbol{\theta} \subseteq[0,2 \pi)$ of angles, defined as

$$
M_{\boldsymbol{\theta}} f(x):=\sup _{R} \frac{1}{|R|} \int_{R}|f|,
$$

where the upper bound extends to all rectangles $R$ in $\mathbf{R}^{2}$ containing $x$, one side of which make an angle $\theta \in \boldsymbol{\theta}$ with the horizontal axis.

It has been shown in 1977 by Cordoba and Fefferman [3] that whenever $\boldsymbol{\theta}=\left\{\theta_{j}\right\}$ is the image of a lacunary sequence (i.e. satisfying $\theta_{j+1} \simeq \lambda \theta_{j}$ for some $0<\lambda<1$ ), then $M_{\boldsymbol{\theta}}$ has weak type $(2,2)$ - and hence is also bounded on $L^{p}\left(\mathbf{R}^{2}\right)$ for all $2 \leqslant p<$ $\infty$. The latter statement was extended to any $1<p<\infty$ a year later by Nagel, Stein and Wainger in [13], and subsequently by Sjögren and Sjölin in [15]. When $\boldsymbol{\theta}=\{0\} \cup\left\{1 / j: j \in \mathbf{N}^{*}\right\}$, de Guzmán proved in 1981 (see [6]) that $M_{\boldsymbol{\theta}}$ is always unbounded on $L^{p}\left(\mathbf{R}^{2}\right)$ for any $p>1$. Later, many authors considered the influence that the "size" of the set $\boldsymbol{\theta}$ has on its yielding the (un)boundedness of $M_{\boldsymbol{\theta}}$ on some $L^{p}$ spaces; a case of particular interest was that of Cantor sets, dealt with by e.g. Katz in 1996 [9] and Hare in 2000 [8]. Lately, in 2009, Bateman gave in [1] a beautiful characterization of sets of angles $\boldsymbol{\theta}$ yielding the boundedness of $M_{\boldsymbol{\theta}}$ in $L^{p}\left(\mathbf{R}^{2}\right)$ for all $1<p<\infty$, showing it is equivalent to the possibility of covering $\boldsymbol{\theta}$ by a finite collection of $N$-lacunary sets (see [1] for a definition). Even more recently in 2013,

https://doi.org/10.5186/aasfm.2020.4523

2010 Mathematics Subject Classification: Primary 42B25; Secondary 26B05.

Key words: Lebesgue's differentiation theorem, rectangular differentiation bases, directional maximal operators. 
Hagelstein studied in [7] the relation between the Minkowski dimension of $\boldsymbol{\theta}$ being zero, and the boundedness in $L^{p}\left(\mathbf{R}^{2}\right)$ of the associated maximal operator; namely he shows that it is a necessary, but not sufficient condition for its boundedness.

On another hand, the boundedness, and weak boundedness on $L^{p}$ of the maximal operator $M_{\boldsymbol{\theta}}$ is also related to differentiation properties of the associated differentiation basis; namely it is classical, given $1<p<\infty$, that $M_{\boldsymbol{\theta}}$ satisfies a weak type $(p, p)$ inequality if and only if Lebesgue's differentiation theorem holds in $L^{p}\left(\mathbf{R}^{2}\right)$ for the associated basis $\mathscr{R}_{\boldsymbol{\theta}}$ of rectangles, one side of which makes an angle $\theta \in \boldsymbol{\theta}$ with the horizontal axis, i.e. if and only the following equality holds for all $f \in L^{p}\left(\mathbf{R}^{2}\right)$ and a.e. $x \in \mathbf{R}^{2}$ :

$$
f(x)=\lim _{\substack{R \ni x \\ \operatorname{diam} R \rightarrow 0}} \frac{1}{|R|} \int_{R}|f|,
$$

where the limit is taken on rectangles $R \in \mathscr{R}_{\boldsymbol{\theta}}$ containing $x$; we then say that the associated basis differentiates $L^{p}\left(\mathbf{R}^{2}\right)$ (see e.g. [5, Chapter III] where it is studied how the differentiation of function spaces by a basis, and the behavior of the associated maximal operator are related in the class of Orlicz spaces). On the other hand, it also follows from [5, Theorem 1.2, p. 69] that $\mathscr{R}_{\boldsymbol{\theta}}$ differentiates $L^{\infty}\left(\mathbf{R}^{2}\right)$ if and only if $M_{\boldsymbol{\theta}}$ satisfies the following condition: for all $\lambda>0$, there exists a constant $0<c_{\lambda}<\infty$ such that one has, for all measurable set $A$

$$
\left|\left\{M_{\boldsymbol{\theta}} \chi_{A}>\lambda\right\}\right| \leqslant c_{\lambda}|A|
$$

such a condition is called a Tauberian condition on the maximal operator $M_{\boldsymbol{\theta}}$. It involves implicitly the halo function associated to the basis $\mathscr{R}_{\boldsymbol{\theta}}$ and defined for $t \geqslant 1$ by

$$
\psi_{\boldsymbol{\theta}}(t):=\sup \left\{\frac{1}{|A|}\left|\left\{M_{\mathscr{R}_{\boldsymbol{\theta}}} \chi_{A}>\frac{1}{t}\right\}\right|: A \text { bounded and measurable, }|A|>0\right\} .
$$

It was a famous open question by de Guzman [5, p. 178] to understand whether knowing that the halo function of a differentiation basis in $\mathbf{R}^{n}$ behaves like $\psi(t) \simeq$ $t^{p}$ implies that the basis in question differentiates $L^{p}\left(\mathbf{R}^{n}\right)$ (or whether a similar statement holds with Orlicz functions). In their paper [2], Berezhnoi and Novikov answered in a negative way this halo problem, after showing that differentiation bases may distinguish Lorentz and Orlicz spaces associated to a given function. It should be pointed out that understanding which Orlicz/Lebesgue spaces a given differentiation basis does or doesn't differentiate is, in general, a question of crucial importance.

It is, in particular, the result of an observation by Strömberg in [20] that for $\boldsymbol{\theta}=\left\{2^{-k}: k \in \mathbf{N}\right\}$, the above version of Lebesgue's differentiation theorem fails in any Orlicz space "larger" than $L \log ^{2} L\left(\mathbf{R}^{2}\right)$, i.e. in any $L^{\Phi}\left(\mathbf{R}^{2}\right)$ where $\Phi$ is an Orlicz function (see below for the precise definition of those term and space) satisfying $\Phi(t)=o\left(t \log _{+}^{2} t\right)$ at $\infty$.

A first observation we make in the current paper is to observe that $\mathscr{R}_{\boldsymbol{\theta}}$ either differentiates all spaces $L^{p}\left(\mathbf{R}^{2}\right)$ for all $1<p \leqslant \infty$, or fails to differentiate any of those spaces - the main point here being that one can take $p=\infty$ in the above alternative (this result has been obtained for decreasing sequences $\theta$ by Hagelstein and Stokolos in [18]). We expose our argument, relying on Bateman's result [1], in section 2.

In the present paper, we also study for various sequences $\left(\theta_{j}\right)$ decreasing to 0 , the differentiation properties of some differentiation bases of rectangles in $\mathbf{R}^{2}$ whose elements have countably many shapes (i.e. for which the ratio between the length of the horizontal and vertical sides belong to a countable set), knowing moreover that 
one of their sizes make an angle $\theta_{j}$ with the horizontal axis for some $j$, in terms of the speed of convergence of the sequence $\left(\theta_{j}\right)$.

More precisely, we prove three theorems of the following structure, for three classes of sequences $\boldsymbol{\theta}=\left(\theta_{j}\right)$ decreasing to 0 , where $\Phi$ is some Orlicz function and $L^{\Phi}\left(\mathbf{R}^{2}\right)$ is the associated Orlicz space (see below for the precise definitions of the terms used here as well as in the following statement).

Theorem 1. There exists a countable family $\mathscr{Q}$ of intervals of the form $[0, L] \times$ $[0, l]$ having the following properties:

(i) the differentiation basis $\mathscr{B}$ of all intervals in $\mathbf{R}^{2}$ whose shape is that of some $Q \in \mathscr{Q}$, differentiates $L^{1}\left(\mathbf{R}^{2}\right)$

(ii) the differentiation basis $\mathscr{B}_{\boldsymbol{\theta}}$ obtained from $\mathscr{B}$ by allowing its elements to rotate of an angle $\theta \in \boldsymbol{\theta}$ around their lower left vertex, fails to differentiate $L^{\Psi}\left(\mathbf{R}^{2}\right)$ for any Orlicz function $\Psi$ satisfying $\Psi=o(\Phi)$ at $\infty$;

(iii) there exists a differentiation basis $\mathscr{B}^{\prime} \subseteq \mathscr{B}_{\boldsymbol{\theta}}$ which differentiates $L^{\Phi}\left(\mathbf{R}^{2}\right)$ but fails to differentiate $L^{\Psi}\left(\mathbf{R}^{2}\right)$ for any Orlicz function $\Psi$ satisfying $\Psi=o(\Phi)$ at $\infty$ (we then say that $\mathscr{B}^{\prime}$ differentiates precisely $L^{\Phi}\left(\mathbf{R}^{2}\right)$ ).

Namely, we consider the three following cases in the previous statement:

(1) $\boldsymbol{\theta}=\left(\theta_{j}\right)$ satisfying $0<\liminf _{j}\left(\theta_{j+1} / \theta_{j}\right) \leqslant \limsup _{j}\left(\theta_{j+1} / \theta_{j}\right)<1-$ in which case the statement above holds for $\Phi(t)=t\left(1+\log _{+} t\right)$ and $L^{\Phi}\left(\mathbf{R}^{2}\right)=$ $L \log L\left(\mathbf{R}^{2}\right)$ (see Theorem 11);

(2) $\boldsymbol{\theta}=\left(\theta_{j}\right)$ satisfying $0<\liminf _{j}\left(\theta_{j+1} / \theta_{j}^{d}\right) \leqslant \limsup _{j}\left(\theta_{j+1} / \theta_{j}^{d}\right)<1$ for some integer $d>1$ - in which case the statement above holds for $\Phi(t)=t(1+$ $\left.\log _{+} \log _{+} t\right)$ and $L^{\Phi}\left(\mathbf{R}^{2}\right)=L \log \log L\left(\mathbf{R}^{2}\right)$ (see Theorem 13);

(3) $\boldsymbol{\theta}=\left(\theta_{j}\right)$ defined by $\theta_{j}=\arctan \left(a^{j^{d}}\right)$ for some $0<a<1$ and $0<d<1-$ in which case the statement above holds for $\Phi(t)=t\left(1+\log _{+}^{1 / d} t\right)$ and $L^{\Phi}\left(\mathbf{R}^{2}\right)=$ $L \log ^{1 / d} L\left(\mathbf{R}^{2}\right)$ (see Theorem 14).

Those results rely on some geometrical preliminaries (detailed in section 3) and on a nice previous work by Stokolos [16] concerning differentiation bases of rectangles in relation to the Orlicz spaces they differentiate. More specifically, in [16], Stokolos constructs, for Orlicz spaces ranging (roughly speaking) between $L \log L\left(\mathbf{R}^{2}\right)$ and $L \log ^{2} L\left(\mathbf{R}^{2}\right)$, differentiation bases of rectangles $\mathscr{B}$ satisfying the following properties:

(1) rectangles in $\mathscr{B}$ have one side forming an angle $2^{-k}$ with the horizontal axis for some $k \in \mathbf{N}$;

(2) $\mathscr{B}$ differentiates precisely $L^{\Phi}\left(\mathbf{R}^{2}\right)$.

Our point, in comparison to Stokolos' result, is here to consider more general sequences $\left(\theta_{j}\right)$ decreasing to 0 , and to see how their convergence speed influences the Orlicz space that one can differentiate using rectangles obtained from intervals enjoying given shapes by rotations of some angle $\theta_{j}$.

The structure of the paper is as follows: after studying some geometrical preliminaries in the spirit of [11], we obtain in section 4 results concerning the Orlicz spaces a differentiation basis of rectangles associated to a given sequence of angles does not differentiate. Combining the results in those two sections with a lemma by Stokolos (see Lemma 2 below), we manage in section 5 to prove the three versions of Theorem 1 stated above. 


\section{Notations and definitions}

1.1. Rectangles. For our purposes, a standard interval in $\mathbf{R}^{2}$ is an interval of the form $Q=[0, L] \times[0, \ell]$. We then let $Q_{+}:=[L / 2, L] \times[0, \ell]$. Given another (not necessarily standard) interval $Q^{\prime}$, we shall say that $Q^{\prime}$ has the same shape as $Q$ in case there exists $a \in \mathbf{R}^{2}$ and $\alpha>0$ such that $Q^{\prime}=a+\alpha Q$-calling shape of an interval the quotient of its horizontal side by its vertical one, this is equivalent to say their shapes are equal. Given a family of standard intervals $\mathscr{Q}$, we then denote by $\mathscr{B}(\mathscr{Q})$ the family of all intervals in $\mathbf{R}^{2}$ having the same shape as some $Q \in \mathscr{Q}$.

For $\theta \in[0,2 \pi)$ we also denote by $r_{\theta}$ the (counterclockwise) rotation of angle $\theta$ around the origin. Given a set $\boldsymbol{\theta} \subseteq[0,2 \pi)$, we then denote by $\mathscr{B}_{\boldsymbol{\theta}}(\mathscr{Q})$ the set of rectangles of the form $r_{\theta} Q$ for some $Q \in \mathscr{B}(\mathscr{Q})$.

1.2 Differentiation bases. A family $\mathscr{B}=\bigcup_{x \in \mathbf{R}^{2}} \mathscr{B}(x)$ of measurable subsets of $\mathbf{R}^{2}$ with positive measure is called a differentiation basis in case the following conditions hold:

(a) for each $x \in \mathbf{R}^{2}$ and each $B \in \mathscr{B}(x)$, one has $x \in B$;

(b) for each $x \in \mathbf{R}^{2}$, one has $\inf \{\operatorname{diam}(B): B \in \mathscr{B}(x)\}=0$.

It is moreover called translation invariant in case one has $\mathscr{B}(x)=x+\mathscr{B}(0)$ for all $x \in \mathbf{R}^{2}$, and homothecy-invariant if it is translation-invariant and if moreover $\alpha \mathscr{B}(0)=\mathscr{B}(0)$ for all $\alpha>0$. We shall also say, finally, that the basis $\mathscr{B}$ enjoys the Buseman-Feller properties in case for any $B \in \mathscr{B}$, the two properties $B \in \mathscr{B}(x)$ and $x \in B$ are equivalent - from now on, we shall see the collections $\mathscr{B}(\mathscr{Q})$ and $\mathscr{B}_{\boldsymbol{\theta}}(\mathscr{Q})$ defined above as differentiation bases enjoying the Buseman-Feller property.

Associated to a differentiation basis $\mathscr{B}$, there is a maximal operator $M_{\mathscr{B}}$ defined by

$$
M_{\mathscr{B}} f(x):=\sup _{B \in \mathscr{B}(x)} \frac{1}{|B|} \int_{B}|f| .
$$

When $\boldsymbol{\theta} \subseteq[0,2 \pi)$ is a set of angles, and when $\mathscr{B}$ is the collection $\mathscr{R}_{\boldsymbol{\theta}}$ of all rectangles in the plane, one side of which makes an angle $\theta \in \boldsymbol{\theta}$ with the horizontal line, we shall briefly denote $M_{\mathscr{B}}$ by $M_{\boldsymbol{\theta}}$.

One also says that a differentiation basis differentiates a function space $X \subseteq$ $L_{\text {loc }}^{1}\left(\mathbf{R}^{2}\right)$ in case for every $f \in X$, the equality

$$
f(x)=\lim _{\substack{\operatorname{diam}_{B \in \mathscr{B}(x)} \rightarrow 0 \\|B|}} \frac{1}{\mid B}|f|
$$

holds for a.e. $x \in \mathbf{R}^{2}$.

1.3. Orlicz spaces. For our purposes, an Orlicz function is a convex, continuous and increasing function $\Phi:[0, \infty) \rightarrow[0, \infty)$ satisfying $\Phi(0)=0$ and $\Phi(t) \rightarrow \infty$ at $\infty$; we say that an Orlicz function $\Phi$ satisfies the $\Delta_{2}$ condition in case there is an absolute constant $K>0$ such that one has $\Phi(2 t) \leqslant K \Phi(t)$ for all sufficiently large $t$. The Orlicz function $\Psi:[0, \infty) \rightarrow[0, \infty)$ defined by $\Psi(s):=\sup \{t|s|-\Phi(t): 0 \leqslant t<\infty\}$ is then called the complementary function to $\Phi$ (a general theory of Orlicz spaces is presented the two monographs by Krasnosel'skii and Rutickii [10] and by Rao and Ren [14]).

Given an Orlicz function $\Phi$, we let $L^{\Phi}\left(\mathbf{R}^{2}\right)$ denote the set of all measurable functions $f$ in $\mathbf{R}^{2}$ for which $\Phi(|f|)$ is integrable (for $\Phi(t)=t^{p}, 1 \leqslant p<\infty$ this yields the usual Lebesgue space $L^{p}\left(\mathbf{R}^{2}\right)$, while for $\Phi(t)=\Phi_{\beta}(t):=t\left(1+\log _{+}{ }^{\beta} t\right)$, with 
$0<\beta$, and for $\Phi(t):=t\left(1+\log _{+} \log _{+} t\right)$ we get the Orlicz spaces $L \log ^{\beta} L\left(\mathbf{R}^{2}\right)$ and $L \log \log L\left(\mathbf{R}^{2}\right)$, respectively).

Given an Orlicz function $\Phi$, recall that a sublinear operator $T$ is said to be of weak type $(\Phi, \Phi)$ in case there exists a constant $C>0$ such that, for all $f \in L^{\Phi}\left(\mathbf{R}^{2}\right)$ and all $\alpha>0$, one has

$$
\left|\left\{x \in \mathbf{R}^{2}: T f(x)>\alpha\right\}\right| \leqslant \int_{\mathbf{R}^{2}} \Phi\left(\frac{|f|}{\alpha}\right) .
$$

Whenever $\Phi(t)=t^{p}$ for $p \geqslant 1$, we shall say that $T$ has weak type $(p, p)$. It is a fact that, for a Buseman-Feller homothecy-invariant differentiation basis $\mathscr{B}$, the following two properties are equivalent for any given Orlicz function $\Phi$ :

(i) $M_{\mathscr{B}}$ is of weak type $(\Phi, \Phi)$;

(ii) $\mathscr{B}$ differentiates $L^{\Phi}\left(\mathbf{R}^{2}\right)$.

The interested reader will find the details of the latter equivalence in [5, Chapter III] (see in particular Remark 4, p. 90).

Finally, given an Orlicz function $\Phi$, we shall say that a differentiation basis $\mathscr{B}$ differentiates exactly $L^{\Phi}\left(\mathbf{R}^{2}\right)$ in cases it differentiates $L^{\Phi}\left(\mathbf{R}^{2}\right)$ but fails to differentiate $L^{\Psi}\left(\mathbf{R}^{2}\right)$ for any Orlicz function $\Psi$ satisfying $\Psi(t)=o(\Phi(t))$ at $\infty$.

1.4. A lemma by Stokolos. The following useful lemma, which is a particular case of [16, Lemma A], will be useful to us in section 5 .

Lemma 2. Assume that $\Phi$ is an Orlicz function satisfying the $\Delta_{2}$ condition and let $\mathscr{R}=\bigcup_{k \in \mathbf{N}} \mathscr{R}_{k}$ where, for each $k \in \mathbf{N}, \mathscr{R}_{k}$ is a finite collection of rectangles in $\mathbf{R}^{2}$. Assume also that there exists a sequence $\left(\lambda_{k}\right)$ increasing to $\infty$ as $k \rightarrow \infty$, and a sequence of balls $B_{k}$ satisfying the following properties:

(i) all members of $\mathscr{R}_{k}$ have equal area;

(ii) for any finite collection $\mathscr{S} \subseteq \mathscr{R}_{k}$, one has

$$
\int_{\mathbf{R}^{2}} \Psi\left(\sum_{R \in \mathscr{S}} \chi_{R}\right) \leqslant c_{1} \sum_{R \in \mathscr{S}}|R|,
$$

where $\Psi$ denotes the complementary function to $\Phi$;

(iii) for any $R \in \mathscr{R}_{k}$, one has

$$
\frac{\left|R \cap B_{k}\right|}{|R|} \geqslant \frac{c_{2}}{\lambda_{n}}
$$

(iv) $\left|\bigcup \mathscr{R}_{k}\right| \geqslant c_{3} \Phi\left(\lambda_{k}\right)\left|E_{k}\right|$;

here, $c_{1}>0, c_{2}>0$ and $c_{3}>0$ are constants. Under those assumptions, there exists a differentiation basis $\mathscr{B}$ satisfying $\mathscr{B} \subseteq \mathscr{B}(\mathscr{R})$ that differentiates precisely $L^{\Phi}\left(\mathbf{R}^{2}\right)$.

Let us now move on to the exposition of our results. Before to discuss the type of statements contained in Theorem 1, let us first formulate our observation concerning the boundedness in $L^{p}, 1<p \leqslant \infty$, of the maximal operator $M_{\boldsymbol{\theta}}$.

\section{Boundedness of the directional maximal operator in $L^{p}$ and $L^{\infty}$ spaces}

We announced the forthcoming proposition in the introduction; the key thing here is that one can include $p=\infty$ in the statement of the "differentiation" dichotomy.

Proposition 3. Let $\boldsymbol{\theta}$ be as above, let $\mathscr{R}_{\boldsymbol{\theta}}$ be the differentiation basis (defined above) of all rectangles in the plane, one side of which makes an angle $\theta \in \boldsymbol{\theta}$ with the 
horizontal line, and let $M_{\boldsymbol{\theta}}$ be the associated maximal operator. Then the following dichotomy holds:

(1) Either $\mathscr{R}_{\boldsymbol{\theta}}$ is bounded on $L^{p}\left(\mathbf{R}^{2}\right)$ for all $1<p<\infty$, in which case the associated differentiation basis $\mathscr{B}$ differentiates all $L^{p}\left(\mathbf{R}^{2}\right), 1<p \leqslant \infty$;

(2) or $M_{\boldsymbol{\theta}}$ is unbounded on all $L^{p}\left(\mathbf{R}^{2}\right), 1<p<\infty$; in this case the associated differentiation basis $\mathscr{B}$ fails to differentiate $L^{p}\left(\mathbf{R}^{2}\right)$ for all $1<p \leqslant \infty$.

Proof. Assuming that $M_{\boldsymbol{\theta}}$ is unbounded on some $L^{p}\left(\mathbf{R}^{2}\right), 1<p<\infty$, it follows from Bateman [1, Theorem 1, p. 56] that $\boldsymbol{\theta}$ admits Kakeya sets, i.e. that for each $N \in \mathbf{N}^{*}$ there exists a collection $\mathscr{R}_{N} \subseteq \mathscr{R}_{\boldsymbol{\theta}}$ verifying

$$
\left|\cup \mathscr{R}_{N}\right| \leqslant \frac{1}{N}\left|\bigcup_{R \in \mathscr{R}_{N}} R^{*}\right|,
$$

where one denoted by $R^{*}$ the rectangle having the same center and width as $R$ but three times its length (we hence assume without loss of generality that none of the $R$ 's is a square).

We show that $M_{\boldsymbol{\theta}}$ is unbounded on all $L^{p}\left(\mathbf{R}^{2}\right)$ by contradiction. Assume thus that there exists some $1<q<\infty$ such that $M_{\boldsymbol{\theta}}$ is bounded on $L^{q}\left(\mathbf{R}^{2}\right)$. This implies in turn that $M_{\boldsymbol{\theta}}$ satisfies a weak-type $(q, q)$ inequality. Since $\mathscr{R}_{\boldsymbol{\theta}}$ is a BusemannFeller differentiation basis invariant with respect to similarities (translations and homothecies), we know, according to e.g. de Guzmán [5, p. 90], that $\mathscr{R}_{\boldsymbol{\theta}}$ would then differentiate $L^{q}\left(\mathbf{R}^{2}\right)$, and hence also $L^{\infty}\left(\mathbf{R}^{2}\right)$. It would then follow from de Guzmán $[5$, Theorem 1.2 , p. 69$]$ that there exists a constant $c>0$ such that for any bounded measurable set $A \subseteq \mathbf{R}^{2}$, one has

$$
\left|\left\{M_{\theta} \chi_{A}>\frac{1}{4}\right\}\right| \leqslant c|A| .
$$

Define, for $N \in \mathbf{N}^{*}, A_{N}:=\bigcup \mathscr{R}_{N}$. Observe that for any $x \in B_{N}:=\bigcup_{R \in \mathscr{R}_{N}} R^{*}$, there exists some $R_{x} \in \mathscr{R}_{N} \subseteq \mathscr{R}_{\boldsymbol{\theta}}$ such that one has $x \in R_{x}^{*}$. Now compute

$$
M_{\boldsymbol{\theta}} \chi_{A_{N}}(x) \geqslant \frac{1}{\left|R_{x}^{*}\right|} \int_{R_{x}^{*}} \chi A_{N} \geqslant \frac{1}{\left|R_{x}^{*}\right|} \int_{R_{x}^{*}} \chi R_{x}=\frac{\left|R_{x} \cap R_{x}^{*}\right|}{\left|R_{x}^{*}\right|}=\frac{1}{3}>\frac{1}{4} .
$$

We hence have $B_{N} \subseteq\left\{M_{\boldsymbol{\theta}} \chi_{A_{N}}>\frac{1}{4}\right\}$; using (1) and (2) we then get

$$
N\left|A_{N}\right| \leqslant\left|B_{N}\right| \leqslant c\left|A_{N}\right|
$$

which is a contradiction for sufficiently large $N$. We conclude that $M_{\boldsymbol{\theta}}$ is unbounded on all $L^{q}\left(\mathbf{R}^{2}\right), 1<q<\infty$, and that $\mathscr{R}_{\boldsymbol{\theta}}$ fails to differentiate $L^{\infty}\left(\mathbf{R}^{n}\right)$.

Remark 4. As pointed out by one of the referees, the preceding proposition has been obtained for decreasing sequences of angles $\boldsymbol{\theta}$ (even though their argument would extend to any set $\boldsymbol{\theta}$ without modification) by Hagelstein and Stokolos in [18]. Instead of relying directly on Bateman's result, it also relies on the beautiful paper by the same authors [19], where they show that if the maximal operator associated to a homothecy-invariant basis of convex sets satisfies a Tauberian condition (which happens in case the basis differentiates $L^{\infty}\left(\mathbf{R}^{n}\right)$ according to de Guzmán's result [5, Theorem 1.2, p. 69]), then it is bounded on $L^{p}\left(\mathbf{R}^{n}\right)$ for sufficiently large $\left.p<\infty\right)$. The argument we presented above uses instead Bateman's geometrical characterization (1) of sets $\boldsymbol{\theta}$ yielding an unbounded maximal operator $M_{\boldsymbol{\theta}}$ in $L^{p}\left(\mathbf{R}^{2}\right), 1<p<\infty$.

We now proceed towards the proof of Theorem 1 by starting with some geometrical preliminaries that were announced in the introduction. 


\section{Some geometrical preliminaries}

The following straightforward geometrical fact is borrowed from [11].

Lemma 5. Fix real numbers $0 \leqslant \vartheta<\theta<\frac{\pi}{2}$ and $0<2 \ell<L$ and let $Q:=$ $[0, L] \times[0, \ell]$. If moreover one has $\tan (\theta-\vartheta) \geqslant 1 / \sqrt{\frac{1}{4}\left(\frac{L}{\ell}\right)^{2}-1}$, then $r_{\vartheta} Q_{+}$and $r_{\theta} Q_{+}$ are disjoint.

Lemma 6. Let $\left\{\theta_{j}\right\} \subset\left[0, \frac{\pi}{4}\right]$ be a decreasing sequence such that, letting $m_{j}:=$ $\tan \theta_{j}$, we have that there exist a constant $C>0$, a constant $0<\zeta<1$ and a sequence $\left\{t_{k}\right\}$ so that, for each $k$, for integers $0 \leqslant j<k$,

$$
m_{j}-m_{k} \geqslant C \zeta^{t_{k}} .
$$

Then, there exist constants $d(C)>c(C)>0$ and $e(C)>0$ depending only on $C$ such that, for each $\varepsilon>0$ and each integer $k \in \mathbf{N}^{\star}=\mathbf{N} \backslash\{0\}$, one can find a standard interval $Q_{k}=\left[0, L_{k}\right] \times\left[0, \ell_{k}\right]$ and a subset $\boldsymbol{\theta}_{k}=\left(\theta_{0}, \ldots, \theta_{k}\right) \subset \boldsymbol{\theta}$ satisfying $\# \boldsymbol{\theta}_{k}=k+1$ and such that the following hold:

(i) $0<2 \ell_{k}<L_{k} \leqslant \varepsilon$;

(ii) $c(C) \zeta^{-t_{2 k}} \leqslant \frac{L_{k}}{\ell_{k}} \leqslant d(C) \zeta^{-t_{2 k}}$;

(iii) $\left|\bigcup_{\theta \in \boldsymbol{\theta}_{k}} r_{\theta} Q_{k}\right| \geqslant \frac{k}{2}\left|Q_{k}\right|$;

(iv) for any subset $\boldsymbol{\theta}^{\prime}=\left(\theta_{i_{0}}, \ldots, \theta_{i_{l}}\right) \subseteq \boldsymbol{\theta}_{k}\left(0 \leqslant l \leqslant k, i_{0}<i_{1}<\cdots<i_{l}\right)$ and any nonnegative, Borel function $\varphi: \mathbf{R}_{+} \rightarrow \mathbf{R}_{+}$satisfying $\varphi(0)=0$, one has

$$
\int_{\mathbf{R}^{2}} \varphi\left(\sum_{\theta \in \boldsymbol{\theta}^{\prime}} \chi_{r_{\theta} Q_{k}}\right) \leqslant e(C)\left|Q_{k}\right| \zeta^{t_{2 k}} \sum_{j=0}^{l} \varphi(j+1) \sum_{r=j}^{l} \zeta^{-t_{i_{r}}} .
$$

Proof. To prove this lemma, observe first that letting $m_{j}:=\tan \theta_{j}$ for all $j \geqslant 0$, we have $m_{j} \leqslant 1$ for all $j$, so that one can compute, for integers $0 \leqslant j<k$

$$
\tan \left(\theta_{j}-\theta_{k}\right)=\frac{m_{j}-m_{k}}{1+m_{j} m_{k}} \geqslant \frac{1}{2}\left(m_{j}-m_{k}\right) \geqslant \frac{C}{2} \zeta^{t_{k}} .
$$

Now choose, for all $k$, real numbers $0<2 l<L \leqslant \varepsilon$ (we write $L$ and $\ell$ instead of $L_{k}$ and $\ell_{k}$ here, for the index $k$ remains constant all through the proof) satisfying

$$
\left(\frac{L}{\ell}\right)^{2}=4+16 C^{-2} \zeta^{-2 t_{2 k}}
$$

It is clear that one has

$$
\frac{L}{\ell}=2 \zeta^{-t_{2 k}} \sqrt{\zeta^{2 t_{2 k}}+4 C^{-2}}
$$

so that (i) and (ii) hold if we take, for example, $c(C):=2 \sqrt{4 C^{-2}}=\frac{4}{C}$ and $d(C):=$ $2 \sqrt{1+4 C^{-2}}$.

In order to show (iii), define $Q:=[0, L] \times[0, \ell]$ and observe that one has

$$
\tan \left(\theta_{j}-\theta_{k}\right) \geqslant \frac{C}{2} \zeta^{t_{2 k}}=\frac{C}{2} \frac{2}{C} \frac{1}{\sqrt{\frac{1}{4}\left(\frac{L}{\ell}\right)^{2}-1}}=\frac{1}{\sqrt{\frac{1}{4}\left(\frac{L}{\ell}\right)^{2}-1}},
$$

for all integers $j<k$ with $k \in \mathbf{N}^{\star}$. According to Lemma 5, this ensures that the family $\left\{r_{\theta_{j}} Q_{+}: j \in \mathbf{N}, 0 \leqslant j \leqslant k\right\}$ consists of pairwise disjoints sets; in particular we 
get

$$
\left|\bigcup_{j=0}^{k} r_{\theta_{j}} Q\right| \geqslant\left|\bigsqcup_{j=0}^{k} r_{\theta_{j}} Q_{+}\right|=k \frac{|Q|}{2}
$$

(we used $\sqcup$ to indicate a disjoint union), which proves (iii).
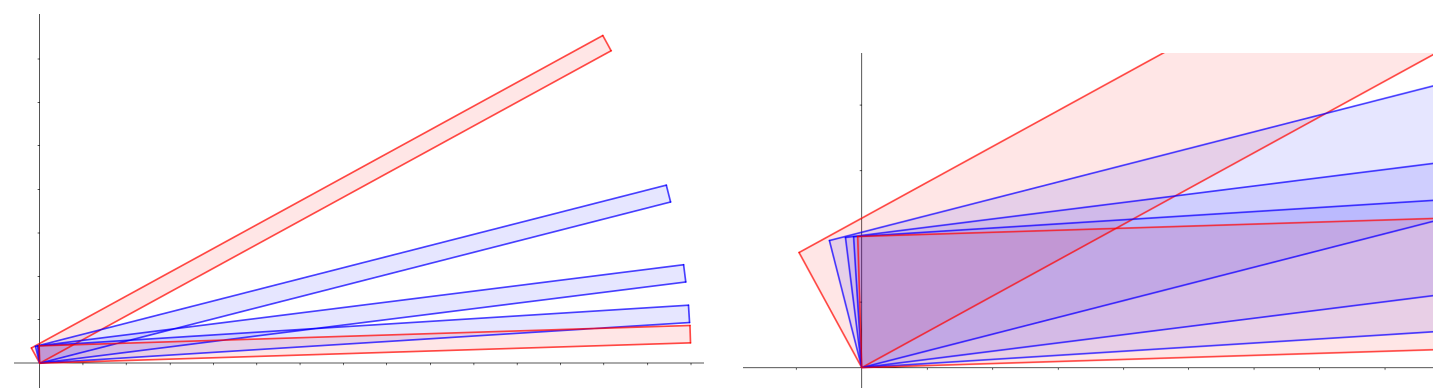

Figure 1. Intersection of rectangles $r_{\alpha_{r_{i}}} Q_{k}, 0 \leqslant i \leqslant p$.

In order to prove (iv), start by writing $\alpha_{r}:=\theta_{i_{r}}$ in order to alleviate notations in the sequel. Given a finite sequence $r_{0}<\cdots<r_{p}=(p \leqslant l)$, observe first that one has (see Figure 1)

$$
\bigcap_{i=0}^{p} r_{\alpha_{r_{i}}} Q_{k}=r_{\alpha_{r_{0}}} Q_{k} \cap r_{\alpha_{r_{p}}} Q_{k}
$$

from which it follows that one has (see Figure 2)

$$
\left|\bigcap_{i=0}^{p} r_{\alpha_{r_{i}}} Q_{k}\right|=\frac{1}{2}|O P| h \leqslant \frac{1}{2} \frac{\ell_{k}}{\sin \hat{P}} \cdot \ell_{k} \geqslant \ell_{k}^{2} \frac{1}{\tan \left(\alpha_{r_{0}}-\alpha_{r_{p}}\right)},
$$

since one has $\sin \hat{P}=\sin \left(\alpha_{r_{0}}-\alpha_{r_{p}}\right)=\tan \left(\alpha_{r_{0}}-\alpha_{r_{p}}\right) \cos \left(\alpha_{r_{0}}-\alpha_{r_{p}}\right) \geqslant \frac{1}{2} \tan \left(\alpha_{r_{0}}-\right.$ $\alpha_{r_{p}}$ ) (recall that $\alpha_{r_{0}}-\alpha_{r_{p}} \leqslant \pi / 4 \leqslant \pi / 3$.

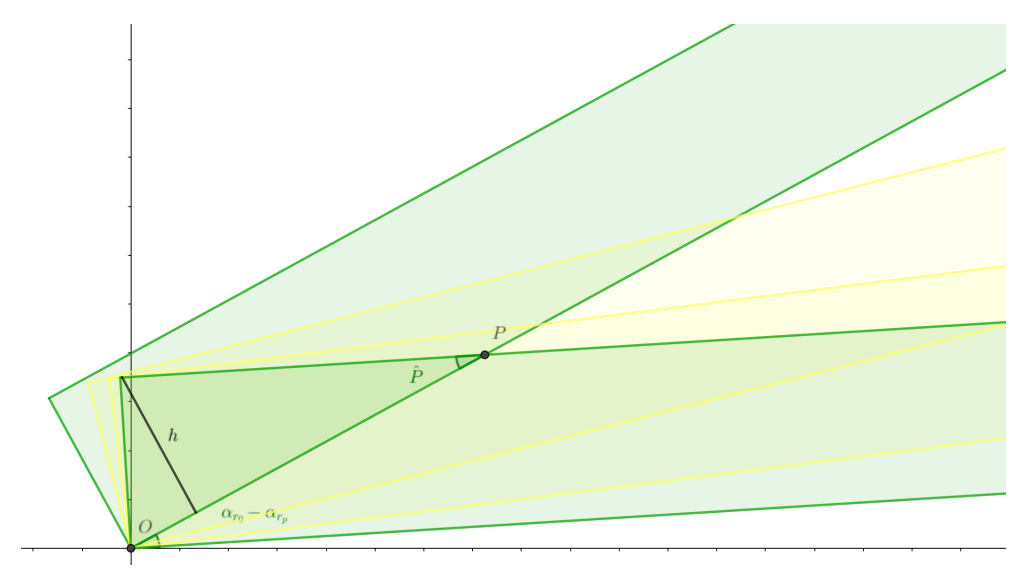

Figure 2. Intersection of rectangles $r_{\alpha_{r_{i}}} Q_{k}, 0 \leqslant i \leqslant p$.

Now let $\chi:=\sum_{\theta \in \boldsymbol{\theta}^{\prime}} \chi_{r_{\theta} Q_{k}}$ and fix $0 \leqslant j \leqslant l$.

Claim 1. One has $|\{\chi=j+1\}| \leqslant \frac{1}{2} \ell_{k}^{2} \sum_{s=0}^{l-j} \frac{1}{\tan \left(\alpha_{s}-\alpha_{s+j}\right)}$.

Proof of the claim. If $x \in \mathbf{R}^{2}$ satisfies $\chi(x)=j+1$, there exists $j+1$ angles $\alpha_{r_{0}}, \ldots, \alpha_{r_{j}}\left(r_{0}<r_{1}<\cdots<r_{j}\right)$ such that one has

$$
x \in \bigcap_{i=0}^{j} r_{\alpha_{r_{i}}} Q_{k}=r_{\alpha_{r_{0}}} Q_{k} \cap r_{\alpha_{r_{j}}} Q_{k}=\bigcap_{r=r_{0}}^{r_{j}} r_{\alpha_{r}} Q_{k} .
$$


Hence if $r_{0}<r_{1}<\cdots<r_{j}$ is not a sequence of successive integers, we have in fact $\chi(x) \geqslant 1+r_{j}-r_{0}>j+1$. This proves that one has

$$
\{\chi=j+1\} \subseteq \bigcup_{s=0}^{l-j} \bigcap_{r=s}^{s+j} r_{\alpha_{r}} Q_{k}=\bigcup_{s=0}^{l-j}\left(r_{\alpha_{s}} Q_{k} \cap r_{\alpha_{s+j}} Q_{k}\right) .
$$

Hence one computes

$$
|\{\chi=j+1\}| \leqslant \ell_{k}^{2} \sum_{s=0}^{l-j} \frac{1}{\tan \left(\alpha_{s}-\alpha_{s+j}\right)},
$$

which proves the claim.

Recalling that one has $\tan \left(\alpha_{s}-\alpha_{s+j}\right)=\tan \left(\theta_{i_{s}}-\theta_{i_{s+j}}\right) \geqslant \frac{C}{2} \zeta^{t_{i_{s+j}}}$ for all $0 \leqslant s \leqslant$ $l-j$, we compute

$$
|\{\chi=j+1\}| \leqslant \frac{2}{C} \ell_{k} L_{k} \frac{\ell_{k}}{L_{k}} \sum_{s=0}^{l-j} \zeta^{-t_{i_{s+j}}} \leqslant \frac{2}{C c(C)}\left|Q_{k}\right| \zeta^{t_{2 k}} \sum_{r=j}^{l} \zeta^{-t_{i_{r}}} .
$$

Letting $e(C):=\frac{2}{C c(C)}$, we finally obtain

$$
\int_{\mathbf{R}^{2}} \varphi(\chi)=\sum_{j=0}^{l} \varphi(j+1)|\{\chi=j+1\}| \leqslant e(C)\left|Q_{k}\right| \zeta^{t_{2 k}} \sum_{j=0}^{l} \varphi(j+1) \sum_{r=j}^{l} \zeta^{-t_{i_{r}}},
$$

which proves (iv) and hence finishes the proof of the lemma.

The next proposition will be useful in order to study the maximal operator $M_{r_{\boldsymbol{\theta}} \mathscr{R}}$.

Proposition 7. Assume that $\boldsymbol{\theta}$ is as in Lemma 6. There exists a family $\mathscr{Q}=$ $\left\{Q_{k}: k \in \mathbf{N}\right\}$ of standard intervals in $\mathbf{R}^{2}$ which is totally ordered by inclusion, satisfies $\inf \{\operatorname{diam} Q: Q \in \mathscr{Q}\}=0$ and satisfies the following property: for any sufficiently large integer $k$, there exist sets $\boldsymbol{\theta}_{k} \subseteq \boldsymbol{\theta}$ and $\Theta_{k} \subseteq \mathbf{R}^{2}$ satisfying $\# \boldsymbol{\theta}_{k}=k+1$ as well as the following conditions (we define $\mathscr{R}_{k}:=\left\{r_{\theta} Q_{k}: \theta \in \boldsymbol{\theta}_{k}\right\}$ and $Y_{k}:=\bigcup \mathscr{R}_{k}$ ):

(i) $\left|Y_{k}\right| \geqslant \gamma(C) k \zeta^{-t_{2 k}}\left|\Theta_{k}\right|$;

(ii) for all $R \in \mathscr{R}_{k}$, one has:

$$
\frac{\left|R \cap \Theta_{k}\right|}{|R|} \geqslant \gamma^{\prime}(C) \zeta^{t_{2 k}}
$$

(iii) all rectangles in $\mathscr{R}_{k}$ have the same area;

(iv) for any subset $\boldsymbol{\theta}^{\prime}=\left(\theta_{i_{0}}, \ldots, \theta_{i_{l}}\right) \subseteq \boldsymbol{\theta}_{k}\left(0 \leqslant l \leqslant k, 0 \leqslant i_{0}<i_{1}<\cdots<i_{l} \leqslant k\right)$ and any nonnegative, Borel function $\varphi: \mathbf{R}_{+} \rightarrow \mathbf{R}_{+}$satisfying $\varphi(0)=0$, one has:

$$
\int_{\mathbf{R}^{2}} \varphi\left(\sum_{\theta \in \boldsymbol{\theta}^{\prime}} \chi_{r_{\theta} Q_{k}}\right) \leqslant \gamma^{\prime \prime}(C)\left|Q_{k}\right| \zeta^{t_{2 k}} \sum_{j=0}^{l} \varphi(j+1) \sum_{r=j}^{l} \zeta^{-t_{i_{r}}}
$$

where $\gamma(C)>0, \gamma^{\prime}(C)>0$ and $\gamma^{\prime \prime}(C)>0$ are constants depending only on $C$.

Proof. Define $\mathscr{R}=\left\{Q_{k}: k \in \mathbf{N}^{*}\right\}$ where the sequence $\left(Q_{k}\right)_{k \in \mathbf{N}^{*}}$ is defined inductively as follows. We choose $Q_{1}=\left[0, L_{1}\right] \times\left[0, \ell_{1}\right]$ and $\boldsymbol{\theta}_{1} \subseteq \boldsymbol{\theta}$ associated to $k=1$ and $\varepsilon=1$ according to Lemma 6 . Assuming that $Q_{1}, \ldots, Q_{k}$ have been constructed, for some integer $k \in \mathbf{N}^{*}$, we choose $Q_{k+1}=\left[0, L_{k+1}\right] \times\left[0, \ell_{k+1}\right]$ and $\boldsymbol{\theta}_{k+1}$ associated to $k+1$ and $\varepsilon=\min \left(\ell_{k}, 1 / k\right)$ according to Lemma 6 . Since the sequence $\left(Q_{k}\right)_{k \in \mathbf{N}^{*}}$ is a nonincreasing sequence of rectangles, it is clear that $\mathscr{Q}$ is totally ordered by inclusion. It is also clear by construction that one has $\inf \left\{\operatorname{diam} Q_{k}: k \in \mathbf{N}\right\}=0$. 
Now fix $k \in \mathbf{N}^{*}$ and define $\Theta_{k}:=B\left(0, \ell_{k}\right)$ and $\mathscr{R}_{k}:=\left\{r_{\theta} Q_{k}: \theta \in \boldsymbol{\theta}_{k}\right\}$. Compute hence, using [Lemma 6, (ii) and (iii)]

$$
\left|Y_{k}\right| \geqslant \frac{k}{2}\left|Q_{k}\right|=\frac{k}{2} L_{k} l_{k}=\frac{1}{2 \pi} k \frac{L_{k}}{l_{k}}\left|\Theta_{k}\right| \geqslant \frac{1}{2 \pi} k c(C) \zeta^{-t_{2 k}}\left|\Theta_{k}\right|
$$

so that (i) is proved with $\gamma(C):=\frac{c(C)}{2 \pi}$.

For any $R \in \mathscr{R}_{k}$, there exists $\theta \in \boldsymbol{\theta}_{k}$ such that one has $R=r_{\theta} Q_{k}$; we hence compute

$$
\frac{\left|R \cap \Theta_{k}\right|}{|R|}=\frac{\left|\Theta_{k} \cap r_{\theta} Q_{k}\right|}{\left|Q_{k}\right|}=\frac{\frac{1}{4} \cdot \pi \ell_{k}^{2}}{L_{k} \ell_{k}}=\frac{\pi}{4} \cdot \frac{\ell_{k}}{L_{k}} \geqslant \frac{\pi}{4} \frac{1}{d(C)} \zeta^{t_{2 k}},
$$

which finishes the proof of (ii) if we set $\gamma^{\prime}(C):=\frac{\pi}{4} \frac{1}{d(C)}$. Now (iii) is clear, while (iv) results immediately from [Lemma 6, (iv)].

Remark 8. In the conditions of the previous proposition, statement (ii) can be reformulated as follows:

(ii') for all $x \in Y_{k}$, one has $M_{\mathscr{B}_{\theta}(\mathscr{Q})} \chi_{\Theta_{k}}(x) \geqslant \gamma^{\prime}(C) \zeta^{t_{2 k}}$.

Using the previous proposition, we can, using standard techniques developed e.g. in a previous work by the second and third authors [12] or in a paper by the two first authors [4], obtain negative differentiation results in a range of Orlicz spaces for some differentiation bases of rectangles associated to various sets $\boldsymbol{\theta}$.

\section{Bad Orlicz spaces for some maximal functions}

In the following statement, we let $\Phi_{\beta}(t):=t\left(1+\log _{+}^{\beta} t\right)$.

Proposition 9. Assume that $\boldsymbol{\theta}$ is as in Lemma 6, that the sequence $t_{k}$ tends to $+\infty$ as $k \rightarrow \infty$ and the sequence $\left\{\frac{t_{k}{ }^{\beta}}{k}\right\}$ is bounded above for some $\beta>0$, that is $M:=\lim \sup _{k} \frac{t_{k}{ }^{\beta}}{k}<\infty$. There exists a (countable) family $\mathscr{Q}$ of standard intervals in $\mathbf{R}^{2}$ with $\inf \{\operatorname{diam} Q: Q \in \mathscr{R}\}=0$, satisfying the following conditions:

(i) $M_{\mathscr{B}(\mathscr{Q})}$ has weak type $(1,1)$, and hence the differentiation basis $\mathscr{B}(\mathscr{Q})$ differentiates $L^{1}\left(\mathbf{R}^{2}\right)$;

(ii) for any Orlicz function $\Psi$ satisfying $\Psi=o\left(\Phi_{\beta}\right)$ at $\infty, M_{\mathscr{B}_{\theta}(\mathscr{Q})}$ fails to be of weak type $(\Psi, \Psi)$, and hence the associated differentiation basis $\mathscr{B}_{\boldsymbol{\theta}}$ fails to differentiate $L^{\Psi}\left(\mathbf{R}^{2}\right)$.

Proof. We keep the notations of Proposition 7 and call $\mathscr{Q}$ the family of rectangles given by Proposition 7 . Observe first that, since $\mathscr{Q}$ is totally ordered by inclusion, it follows e.g. from [17, Claim 1] that $M_{\mathscr{B}(\mathscr{Q})}$ satisfies a weak $(1,1)$ inequality.

In order to show (ii), define, for $k$ sufficiently large, $f_{k}:=\frac{1}{\gamma^{\prime}(C)} \cdot \zeta^{-t_{2 k}} \chi_{\Theta_{k}}$, where $\Theta_{k}$ and $Y_{k}$ are associated to $k$ and $\mathscr{Q}$ according to Proposition 7.

Claim 2. For each sufficiently large $k$, we have

$$
\left|\left\{x \in \mathbf{R}^{2}: M_{\mathscr{B}_{\boldsymbol{\theta}}(\mathscr{Q})} f_{k}(x) \geqslant 1\right\}\right| \geqslant \gamma_{1}(\beta, C, \zeta, M) \int_{\mathbf{R}^{2}} \Phi_{\beta}\left(f_{k}\right) .
$$

Proof of the claim. To prove this claim, one observes that for $x \in Y_{k}$ we have $M_{\mathscr{B}_{\boldsymbol{\theta}}(\mathscr{Q})} f_{k}(x) \geqslant 1$ according to (ii') in Remark 8 . Yet, on the other hand, one 
computes, for $k$ sufficiently large

$$
\begin{aligned}
\int_{\mathbf{R}^{2}} \Phi_{\beta}\left(f_{k}\right) & \leqslant \frac{1}{\gamma^{\prime}(C)} \cdot \zeta^{-t_{2 k}}\left|\Theta_{k}\right|\left(2 t_{2 k} \log \frac{1}{\zeta}\right)^{\beta} \\
& \leqslant \frac{1}{\gamma(C) \gamma^{\prime}(C)}\left|Y_{k}\right|(2 k)^{-1}\left(2 t_{2 k} \log \frac{1}{\zeta}\right)^{\beta} \\
& <2^{\beta+1} \tilde{M} \frac{1}{\gamma(C) \gamma^{\prime}(C)}\left|Y_{k}\right|\left(\log \frac{1}{\zeta}\right)^{\beta}
\end{aligned}
$$

where $\tilde{M}:=\max \{1, M\}$ and

$$
\gamma_{1}(\beta, C, \zeta, M):=\left(\log \frac{1}{\zeta}\right)^{-\beta}\left[\frac{\gamma^{\prime}(C) \gamma(C)}{2^{\beta+1} \tilde{M}}\right] .
$$

The claim follows.

Claim 3. For any $\Phi$ satisfying $\Phi=o\left(\Phi_{\beta}\right)$ at $\infty$ and for each constant $T>0$, we have:

$$
\lim _{k \rightarrow \infty} \frac{\int_{\mathbf{R}^{2}} \Phi_{\beta}\left(\left|f_{k}\right|\right)}{\int_{\mathbf{R}^{2}} \Phi\left(T\left|f_{k}\right|\right)}=\infty .
$$

Proof of the claim. Compute for any $k$,

$$
\frac{\int_{\mathbf{R}^{2}} \Phi\left(T\left|f_{k}\right|\right)}{\int_{\mathbf{R}^{2}} \Phi_{\beta}\left(\left|f_{k}\right|\right)}=\frac{\Phi\left(\zeta^{-t_{2 k}} T / \gamma^{\prime}(C)\right)}{\Phi_{\beta}\left(\zeta^{-t_{2 k}} / \gamma^{\prime}(C)\right)}=\frac{\Phi\left(\zeta^{-t_{2 k}} T / \gamma^{\prime}(C)\right)}{\Phi_{\beta}\left(\zeta^{-t_{2 k}} T / \gamma^{\prime}(C)\right)} \frac{\Phi_{\beta}\left(\zeta^{-t_{2 k}} T / \gamma^{\prime}(C)\right)}{\Phi_{\beta}\left(\zeta^{-t_{2 k}} / \gamma^{\prime}(C)\right)}
$$

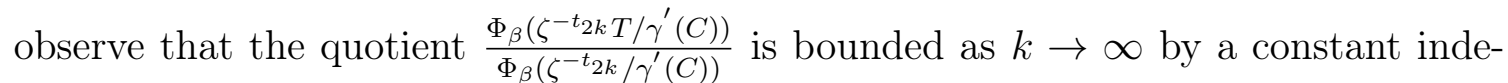
pendent of $k$, and observe that by assumption $\frac{\Phi\left(\zeta^{-t_{2 k}} T / \gamma^{\prime}(C)\right)}{\Phi_{\beta}\left(\zeta^{\left.-t_{2 k} T / \gamma^{\prime}(C)\right)}\right.}$ tends to zero as $k \rightarrow \infty$. The claim is proved.

We now finish the proof of Proposition 9. To this purpose, fix $\Phi$ an Orlicz function satisfying $\Phi=o\left(\Phi_{\beta}\right)$ at $\infty$ and assume that there exists a constant $T>0$ such that, for any $\alpha>0$, one has

$$
\left|\left\{x \in \mathbf{R}^{2}: M_{\mathscr{B}_{\boldsymbol{\theta}}(\mathscr{Q})} f(x)>\alpha\right\}\right| \leqslant \int_{\mathbf{R}^{2}} \Phi\left(\frac{T|f|}{\alpha}\right) .
$$

Using Claim 2, we would then get, for each $k$ sufficiently large

$$
0<\gamma_{1}(\beta, C, \zeta, M) \int_{\mathbf{R}^{2}} \Phi_{\beta}\left(f_{k}\right) \leqslant\left|\left\{x \in \mathbf{R}^{2}: M_{\mathscr{B}_{\boldsymbol{\theta}}(\mathscr{Q})} f_{k}(x)>\frac{1}{2}\right\}\right| \leqslant \int_{\mathbf{R}^{n}} \Phi\left(2 T f_{k}\right),
$$

contradicting the previous claim and proving the theorem.

The following result is proved in a very similar way and we omit the proof.

Proposition 10. Assume that $\boldsymbol{\theta}$ is as in Lemma 6, that the sequence $t_{k}$ tends to $+\infty$ as $k \rightarrow \infty$, that it satisfies $M:=\limsup _{k} \frac{\log t_{k}}{k}<\infty$ and define $\Phi(t)=$ $t\left(1+\log _{+} \log _{+} t\right)$. There exists a (countable) family $\mathscr{Q}$ of standard intervals in $\mathbf{R}^{2}$ with $\inf \{\operatorname{diam} Q: Q \in \mathscr{Q}\}=0$, satisfying the following conditions:

(i) $M_{\mathscr{B}(\mathscr{Q})}$ has weak type $(1,1)$, and hence the associated differentiation basis $\mathscr{B}(\mathscr{Q})$ differentiates $L^{1}\left(\mathbf{R}^{2}\right)$

(ii) for any Orlicz function $\Psi$ satisfying $\Psi=o(\Phi)$ at $\infty, M_{\mathscr{B}_{\boldsymbol{\theta}}(\mathscr{Q})}$ fails to be of weak type $(\Psi, \Psi)$ - hence the differentiation basis $\mathscr{B}_{\boldsymbol{\theta}}(\mathscr{Q})$ fails to differentiate $L \log \log L\left(\mathbf{R}^{2}\right)$. 
We now turn to proving the three versions of Theorem 1 we stated in the introduction.

\section{Three examples of sequences yielding Theorem 1}

Let $d \in \mathbf{N}$ be fixed. Assume that the sequence $\left(\theta_{k}\right)_{k \in \mathbf{N}} \subseteq(0, \pi / 4]$ is such that one has:

$$
0<\lambda<\liminf _{j \rightarrow \infty} \frac{\theta_{j+1}}{\theta_{j}{ }^{d}} \leqslant \limsup _{j \rightarrow \infty} \frac{\theta_{j+1}}{{\theta_{j}}^{d}}<\mu<1 .
$$

Letting $m_{j}:=\tan \theta_{j}$ for all $j \in \mathbf{N}$, one clearly has:

$$
\lim _{j \rightarrow \infty} \frac{m_{j}}{\theta_{j}}=1
$$

so that (3) also holds for the sequence $\left(m_{j}\right)_{j \in \mathbf{N}}$. There hence exists an index $j_{0} \in \mathbf{N}$ such that, for all $j \geqslant j_{0}$, one has $\lambda \leqslant \frac{m_{j+1}}{m_{j}{ }^{d}} \leqslant \mu$ (we may also and will assume that one has $\left.\frac{\lambda}{2} \leqslant m_{j_{0}} \leqslant \lambda\right)$. For the sake of clarity, we shall now consider that $j_{0}=0$ and compute, for an integer $0 \leqslant j<k$ :

$$
\tan \left(\theta_{j}-\theta_{k}\right)=\frac{m_{j}-m_{k}}{1+m_{j} m_{k}} \geqslant \frac{1}{2}\left(m_{j}-m_{k}\right) .
$$

We also have, for every integer $0 \leqslant j<k$,

$$
\lambda^{\sum_{i=0}^{k-j-1} d^{i}} m_{j} d^{k-j} \leqslant m_{k} \leqslant \mu^{\sum_{i=0}^{k-j-1} d^{i}} m_{j} d^{k-j} .
$$

5.1. Assume first that $\boldsymbol{d}=1$. Arguing as in [11], we then get

$$
\tan \left(\theta_{j}-\theta_{k}\right) \geqslant \frac{1}{2} m_{0} \cdot \lambda^{k} \cdot\left[\mu^{-1}-1\right],
$$

so that if we set $C=\frac{1}{2} m_{0} \cdot\left[\mu^{-1}-1\right], \zeta=\lambda, t_{k}=k$ and take $\beta=1$ then the hypotheses of Proposition 9 are satisfied. Let $\mathscr{Q}, \mathscr{R}_{k}$ and $\boldsymbol{\theta}_{k}$ be associated to $\boldsymbol{\theta}$ by Proposition 7 ; define $\Phi(t):=t\left(1+\log _{+} t\right)$ and observe that it is easy to see (see e.g. [10, Chapter 1]) that one can have $\Psi(t) \leqslant K_{1} e^{t}$ for the complementary function $\Psi$ to $\Phi$. There is no loss of generality, of course, to assume $\zeta<1 / e$.

Now fix a subset $\boldsymbol{\theta}^{\prime}=\left\{\theta_{i_{r}}: 0 \leqslant r \leqslant l\right\} \subset \boldsymbol{\theta}_{k}\left(0 \leqslant i_{0}<i_{1}<\cdots<i_{l} \leqslant k\right)$ and write, using $\varphi=\Psi$ in [Proposition 7, (iv)],

$$
\int_{\mathbf{R}^{2}} \Psi\left(\sum_{\theta \in \boldsymbol{\theta}^{\prime}} \chi_{r_{\theta} Q_{k}}\right) \leqslant K_{1}^{\prime}\left|Q_{k}\right| \zeta^{2 k} \sum_{j=0}^{l} e^{j} \sum_{r=j}^{l} \zeta^{-i_{r}}
$$

where $K_{1}^{\prime}=e K_{1} e(C)>0$. Write then

$$
\sum_{r=j}^{l} \zeta^{-i_{r}} \leqslant \zeta^{-i_{l}} \sum_{s=0}^{\infty} \zeta^{s} \leqslant \frac{\zeta^{-k}}{1-\zeta}
$$

On the other hand one computes

$$
\sum_{j=0}^{l} e^{j}=e^{l} \sum_{j=0}^{l} e^{j-l} \leqslant e^{k} \sum_{s=0}^{\infty} e^{-s} \leqslant \frac{e^{k+1}}{e-1} .
$$

Hence we obtain

$$
\int_{\mathbf{R}^{2}} \Psi\left(\sum_{\theta \in \boldsymbol{\theta}^{\prime}} \chi_{r_{\theta} Q_{k}}\right) \leqslant K_{1}^{\prime}\left|Q_{k}\right| \zeta^{2 k} \cdot \frac{e^{k+1}}{e-1} \cdot \frac{\zeta^{-k}}{1-\zeta} \leqslant \frac{2 K_{1}^{\prime}}{1-\zeta}\left|Q_{k}\right|
$$


since one has $e \zeta<1$ and $e /(e-1) \leqslant 2$.

Combining now Proposition 9 and Lemma 2 for the particular sequence considered in this section, we get the following result.

Theorem 11. Assume that the sequence $\left(\theta_{k}\right)_{k \in \mathbf{N}} \subseteq(0, \pi / 4]$ is such that one has

$$
0<\lambda<\liminf _{j \rightarrow \infty} \frac{\theta_{j+1}}{\theta_{j}} \leqslant \limsup _{j \rightarrow \infty} \frac{\theta_{j+1}}{\theta_{j}}<\mu<1 .
$$

Under those assumptions, there exists a countable family of standard intervals in $\mathbf{R}^{2}$ denoted by $\mathscr{Q}$ and totally ordered by inclusion, satisfying the following properties (where one defines $\Phi(t):=t\left(1+\log _{+} t\right)$ ):

(i) the associated differentiation basis $\mathscr{B}(\mathscr{Q})$ differentiates $L^{1}\left(\mathbf{R}^{2}\right)$;

(ii) for any Orlicz function $\Psi$ satisfying $\Psi=o(\Phi)$ at $\infty$, the differentiation basis $\mathscr{B}_{\boldsymbol{\theta}}(\mathscr{Q})$ fails to differentiate $L^{\Psi}\left(\mathbf{R}^{2}\right)$

(iii) there exists a differentiation basis $\mathscr{B} \subseteq \mathscr{B}_{\boldsymbol{\theta}}(\mathscr{Q})$ that differentiates exactly $L \log L\left(\mathbf{R}^{2}\right)$.

Remark 12. Statement (iii) above is weaker than stating that the basis $\mathscr{B}_{\boldsymbol{\theta}}(\mathscr{Q})$ itself does differentiate (exactly) $L \log L\left(\mathbf{R}^{2}\right)$; however, it is not known to us whether this statement holds or not.

5.2. Assume now that $\boldsymbol{d}>1$ in (3). Using (4), we obtain for $0 \leqslant j<k$

$$
\lambda^{\frac{d^{k-j}-1}{d-1}} m_{j}{ }^{d^{k-j}}=\lambda^{\sum_{i=0}^{k-j-1} d^{i}} m_{j}^{d^{k-j}} \leqslant m_{k} \leqslant \mu^{\sum_{i=0}^{k-j-1} d^{i}} m_{j}{ }^{k-j}=\mu^{\frac{d^{k-j}-1}{d-1}} m_{j} d^{k-j} .
$$

We then compute, for the same $j<k$,

$$
\begin{aligned}
\tan \left(\theta_{j}-\theta_{k}\right) & \geqslant \frac{1}{2}\left(m_{j}-m_{k}\right) \geqslant \frac{1}{2}\left[m_{k} \frac{1}{d^{k-j}} \cdot \mu^{-\frac{\sum_{i=0}^{k-j-1} d^{i}}{d^{k-j}}}-m_{k}\right] \\
& \geqslant \frac{1}{2} m_{k} \cdot\left[\mu^{-\frac{\sum_{i=0}^{k-1} d^{i}}{d^{k-j}}}-1\right] \geqslant \frac{1}{2} m_{0} d^{k} \cdot \lambda^{\sum_{i=0}^{k-1} d^{i}} \cdot\left[\mu^{-\frac{1}{d-1}}-1\right] \\
& =\frac{1}{2} m_{0} d^{k} \cdot \lambda^{d^{k}} \cdot\left[\mu^{-\frac{1}{d-1}}-1\right] \geqslant \frac{1}{2} m_{0} d^{k} \cdot \lambda^{d^{k}} \cdot\left[\mu^{-\frac{1}{d-1}}-1\right] \\
& \geqslant \frac{1}{2}\left(\frac{\lambda}{2}\right)^{d^{k}} \cdot \lambda^{d^{k}} \cdot\left[\mu^{-\frac{1}{d-1}}-1\right] \geqslant \frac{1}{2}\left(\frac{\lambda}{2}\right)^{2 d^{k}} \cdot\left[\mu^{-\frac{1}{d-1}}-1\right] .
\end{aligned}
$$

So if we set $C=\frac{1}{2}\left[\mu^{-\frac{1}{d-1}}-1\right], \zeta=\left(\frac{\lambda}{2}\right)^{2}$ and $t_{k}=d^{k}$, then the hypotheses of Proposition 10 are satisfied. Let hence $\mathscr{Q}, \mathscr{R}_{k}$ and $\boldsymbol{\theta}_{k}$ be associated to $\boldsymbol{\theta}$ by Proposition 7 and define $\Phi(t):=t\left(1+\log _{+} \log _{+} t\right)$ and observe that it is easy to see (see e.g. [10, Chapter 1]) that one can have $\Psi(t) \leqslant K_{2} \exp (\exp t)$ for the complementary function $\Psi$ to $\Phi$. There is no loss of generality, again, to assume $\zeta<1 / e$.

Fix as before a subset $\boldsymbol{\theta}^{\prime}=\left\{\theta_{i_{r}}: 0 \leqslant r \leqslant l\right\} \subset \boldsymbol{\theta}_{k}\left(0 \leqslant i_{0}<i_{1}<\cdots<i_{l} \leqslant k\right)$ and write, using $\varphi=\Psi$ in [Proposition 7 , (iv)]:

$$
\int_{\mathbf{R}^{2}} \Psi\left(\sum_{\theta \in \boldsymbol{\theta}^{\prime}} \chi_{r_{\theta} Q_{k}}\right) \leqslant K_{2}^{\prime}\left|Q_{k}\right| \zeta^{\zeta^{2 k}} \sum_{j=0}^{l} e^{e^{j+1}} \sum_{r=j}^{l} \zeta^{-d^{i r}},
$$

where $K_{2}^{\prime}=K_{2} e(C)>0$. Write then

$$
\sum_{r=j}^{l} \zeta^{-d^{i_{r}}} \leqslant \zeta^{-d^{i} l} \sum_{s=0}^{\infty} \zeta^{s} \leqslant \frac{\zeta^{-d^{k}}}{1-\zeta} .
$$


On the other hand one computes

$$
\sum_{j=0}^{l} e^{e^{j+1}} \leqslant e^{e^{l+1}} \sum_{s=0}^{\infty} e^{-s} \leqslant \frac{e^{1+e^{k+1}}}{e-1} .
$$

Hence we obtain

$$
\begin{aligned}
\int_{\mathbf{R}^{2}} \Psi\left(\sum_{\theta \in \boldsymbol{\theta}^{\prime}} \chi_{r_{\theta} Q_{k}}\right) & \leqslant K_{2}^{\prime} e\left|Q_{k}\right| \zeta^{d^{2 k}} \cdot \frac{e^{e^{k+1}}}{e-1} \cdot \frac{\zeta^{-d^{k}}}{1-\zeta} \\
& \leqslant \frac{K_{2}^{\prime} e}{(1-\zeta)(e-1)}\left|Q_{k}\right| \exp \left[e^{k+1}+d^{k}-\left(d^{2}\right)^{k}\right],
\end{aligned}
$$

since one has $e \zeta<1$. Observing e.g. that $d^{2} \geqslant 4>e$ and that one hence has

$$
e^{k+1}+d^{k}-\left(d^{2}\right)^{k}=e\left[e^{k}-\frac{1}{2 e}\left(d^{2}\right)^{k}\right]+d^{k}-\frac{1}{2}\left(d^{2}\right)^{k},
$$

and since moreover it is clear that $a^{k}-\varepsilon b^{k}$ tends to $-\infty$ as $k \rightarrow \infty$ for any real numbers $1<a<b$ and $\varepsilon>0$, we finally get

$$
\lim _{k \rightarrow \infty} \exp \left[e^{k+1}+d^{k}-\left(d^{2}\right)^{k}\right]=0 .
$$

There thus exists a constant $K_{2}^{\prime \prime}>0$ (depending only on $\boldsymbol{\theta}$ and $d$ ) for which one has

$$
\int_{\mathbf{R}^{2}} \Psi\left(\sum_{\theta \in \boldsymbol{\theta}^{\prime}} \chi_{r_{\theta} Q_{k}}\right) \leqslant K_{2}^{\prime \prime}\left|Q_{k}\right| .
$$

Combining now as above Proposition 10 and Lemma 2 for the particular case of a sequence satisfying (3) for $d>1$, we obtain the following result.

Theorem 13. Assume that $d \in \mathbf{N}^{*}$ and the sequence $\left(\theta_{k}\right)_{k \in \mathbf{N}} \subseteq(0, \pi / 4]$ are such that one has

$$
0<\lambda<\liminf _{j \rightarrow \infty} \frac{\theta_{j+1}}{\theta_{j}^{d}} \leqslant \limsup _{j \rightarrow \infty} \frac{\theta_{j+1}}{\theta_{j}^{d}}<\mu<1
$$

Under those assumptions, there exists a countable family of standard intervals in $\mathbf{R}^{2}$ denoted by $\mathscr{Q}$ and totally ordered by inclusion, satisfying the following properties (where one defines $\Phi(t):=t\left(1+\log _{+} \log _{+} t\right)$ ):

(i) the associated differentiation basis $\mathscr{B}(\mathscr{Q})$ differentiates $L^{1}\left(\mathbf{R}^{2}\right)$;

(ii) for any Orlicz function $\Psi$ satisfying $\Psi=o(\Phi)$ at $\infty$, the differentiation basis $\mathscr{B}_{\boldsymbol{\theta}}(\mathscr{Q})$ fails to differentiate $L^{\Psi}\left(\mathbf{R}^{2}\right)$;

(iii) there exists a differentiation basis $\mathscr{B} \subseteq \mathscr{B}_{\boldsymbol{\theta}}(\mathscr{Q})$ that differentiates exactly $L \log \log L\left(\mathbf{R}^{2}\right)$.

5.3. A non-lacunary example. Let $\left(a_{j}\right)$ be a nonincreasing sequence of positive real numbers with $0<a=\inf _{j} a_{j} \leqslant \sup _{j} a_{j}=b<1$. Clearly, $a_{0}=\max a_{j}=$ $b$. In the sequel we fix a real number $0<d<1$ and we define a sequence $\left(\theta_{j}\right)$ by

$$
\theta_{j}:=\arctan \left[\left(a_{j}\right)^{j^{d}}\right] .
$$


Observe that letting $m_{j}:=\tan \theta_{j}$ for all $j$, we can write, for $j_{0} \leqslant j \leqslant k$,

$$
\begin{aligned}
m_{j}-m_{k} & =\left(a_{j}\right)^{j^{d}}-\left(a_{k}\right)^{k^{d}} \geqslant\left(a_{k}\right)^{j^{d}}-\left(a_{k}\right)^{k^{d}}=\left(a_{k}\right)^{k^{d}}\left[\left(a_{k}\right)^{j^{d}-k^{d}}-1\right] \\
& \geqslant a^{k^{d}}\left[\left(\frac{1}{b}\right)^{k^{d}-j^{d}}-1\right] \geqslant a^{k^{d}}\left[\left(\frac{1}{b}\right)^{k^{d}-(k-1)^{d}}-1\right] .
\end{aligned}
$$

It hence follows in particular that $\left(m_{j}\right)$ (and hence also $\left(\theta_{j}\right)$ ) is a decreasing sequence.

On the other hand, it is easy to observe that one has, for all $j$,

$$
j^{d}-(j-1)^{d}=d j^{d-1}+O\left(j^{d-2}\right),
$$

we get

$$
\left[j^{d}-(j-1)^{d}\right] j^{1-d}=d+O\left(j^{-1}\right),
$$

so that, for $j$ sufficiently large, we have

$$
j^{d}-(j-1)^{d} \geqslant \frac{d}{2 j^{1-d}} .
$$

It is also the case that for $j$ sufficiently large, we always have

$$
\frac{2}{d} j^{1-d} \leqslant \frac{1}{2} b^{-j^{d}}
$$

we shall hence assume that both (6) and (7) hold for $j \geqslant j_{0}$, and we shall, from now on, work with the sequence $\left(\theta_{j}\right)_{j \geqslant j_{0}}$; we also define $\boldsymbol{\theta}:=\left\{\theta_{j}: j \geqslant j_{0}\right\}$.

Now given $k>j_{0}$, we obtain from (5), for all $\max \left\{j_{0}, \log _{a}\left(\log _{a} e\right)\right\} \leqslant j_{1} \leqslant j \leqslant k$, using the inequality $a^{x} \geqslant 1+x$ for all $x \geqslant \log _{a}\left(\log _{a} e\right)$ and then $(6)$ and (7):

$$
m_{j}-m_{k} \geqslant a^{k^{d}}\left[\left(\frac{1}{b}\right)^{k^{d}-(k-1)^{d}}-1\right] \geqslant a^{k^{d}} \frac{d}{2 k^{1-d}} \geqslant 2(a b)^{k^{d}} .
$$

So if we take $C=2, \zeta=a b, t_{k}=k^{d}$ and $\beta=\frac{1}{d}$, we have that the hypotheses of Proposition 9 are satisfied. Let also $\mathscr{Q}, \mathscr{R}_{k}$ and $\boldsymbol{\theta}_{k}$ be associated to $\boldsymbol{\theta}$ by Proposition 7; define now $\Phi(t):=t\left(1+\log _{+}^{1 / d} t\right)$ and observe that it is easy to see (see e.g. [10, Chapter 1]) that one can have $\Psi(t) \leqslant K_{3} e^{t^{d}}$ for the complementary function $\Psi$ to $\Phi$. There is no loss of generality, reducing $\zeta$ if necessary, to assume that one has $\eta:=e \zeta^{2^{d}-1}<1$.

Now fix a subset $\boldsymbol{\theta}^{\prime}=\left\{\theta_{i_{r}}: 0 \leqslant r \leqslant l\right\} \subset \boldsymbol{\theta}_{k}\left(0 \leqslant i_{0}<i_{1}<\cdots<i_{l} \leqslant k\right)$ and write, using $\varphi=\Psi$ in [Proposition 7 , (iv)],

$$
\int_{\mathbf{R}^{2}} \Psi\left(\sum_{\theta \in \boldsymbol{\theta}^{\prime}} \chi_{r_{\theta} Q_{k}}\right) \leqslant K_{3}^{\prime}\left|Q_{k}\right| \zeta^{(2 k)^{d}} \sum_{j=0}^{l} e^{(j+1)^{d}} \sum_{r=j}^{l} \zeta^{-i_{r}^{d}},
$$

where $K_{3}^{\prime}=K_{3} e(C)>0$. Write then

$$
\sum_{r=j}^{l} \zeta^{-i_{r}^{d}} \leqslant \zeta^{-i_{l}^{d}} \sum_{r=j}^{l} \zeta^{i_{l}^{d}-i_{r}^{d}} \leqslant(k+1) \zeta^{-k^{d}}
$$

since we have $\zeta_{l}^{i_{l}^{d}-i_{r}^{d}} \leqslant 1$ for all $j \leqslant r \leqslant l$. On the other hand one computes in a similar fashion

$$
\sum_{j=0}^{l} e^{j^{d}}=e^{l^{d}} \sum_{j=0}^{l} e^{j^{d}-l^{d}} \leqslant(k+1) e^{k^{d}}
$$


Hence we obtain

$$
\int_{\mathbf{R}^{2}} \Psi\left(\sum_{\theta \in \boldsymbol{\theta}^{\prime}} \chi_{r_{\theta} Q_{k}}\right) \leqslant K_{3}^{\prime}\left|Q_{k}\right|(k+1)^{2} \zeta^{(2 k)^{d}-k^{d}} e^{k^{d}}
$$

Yet one has

$$
(k+1)^{2} \zeta^{(2 k)^{d}-k^{d}} e^{k^{d}}=(k+1)^{2}\left(e \zeta^{2^{d}-1}\right)^{k^{d}}=(k+1)^{2} \eta^{k^{d}} \rightarrow 0, \quad k \rightarrow \infty .
$$

Hence there exists a constant $K_{3}^{\prime \prime}>0$, independent of $k$, for which one has

$$
\int_{\mathbf{R}^{2}} \Psi\left(\sum_{\theta \in \boldsymbol{\theta}^{\prime}} \chi_{r_{\theta} Q_{k}}\right) \leqslant K_{3}^{\prime \prime}\left|Q_{k}\right|
$$

Combining now Proposition 9 and Lemma 2 for the particular sequence considered in this section, we get the following result.

Theorem 14. Assume that $0<d<1$ is a real number. Let $\left(a_{j}\right)$ be a nonincreasing sequence of positive real numbers with $0<\inf _{j} a_{j} \leqslant \sup _{j} a_{j}<1$ and define a sequence $\left(\theta_{j}\right)$ by

$$
\theta_{j}:=\arctan \left[\left(a_{j}\right)^{j^{d}}\right] .
$$

Under those assumptions, there exists a countable family of standard intervals in $\mathbf{R}^{2}$ denoted by $\mathscr{Q}$ and totally ordered by inclusion, satisfying the following properties (we define $\Phi(t):=t\left(1+\log _{+}^{1 / d} t\right.$ ) for $\left.\beta>0\right)$ :

(i) the associated differentiation basis $\mathscr{B}(\mathscr{R})$ differentiates $L^{1}\left(\mathbf{R}^{2}\right)$;

(ii) for any Orlicz function $\Psi$ satisfying $\Psi=o(\Phi)$ at $\infty$, the differentiation basis $\mathscr{B}_{\boldsymbol{\theta}}(\mathscr{Q})$ fails to differentiate $L^{\Psi}\left(\mathbf{R}^{2}\right)$;

(iii) there exists a differentiation basis $\mathscr{B} \subseteq \mathscr{B}_{\boldsymbol{\theta}}(\mathscr{Q})$ that differentiates exactly $L \log ^{\frac{1}{d}} L\left(\mathbf{R}^{2}\right)$-hence it also differentiates $L^{p}\left(\mathbf{R}^{2}\right)$ for all $p>1$.

Remark 15. Let $\left(a_{j}\right)$ and $d$ be as above, that is let $\left\{a_{j}\right\}$ be a non-increasing sequence of positive real numbers with $0<a=\inf _{j} a_{j} \leqslant \sup _{j} a_{j}=b<1$ and $0<d<1$. Then $\left(a_{j}\right)^{j^{d}}$ cannot be lacunary according to the definition in [11]. Assume, by contradiction that there exist $\alpha$ and $\beta$ satisfying

$$
0<\alpha \leqslant \frac{a_{j+1}{ }^{(j+1)^{d}}}{a_{j}^{j^{d}}}<\beta<1 .
$$

Then, for each $j \geqslant 2$, we have

$$
\alpha^{\frac{j-1}{j^{d}}} a_{1}^{\frac{1}{j^{d}}} \leqslant a_{j} \leqslant \beta^{\frac{j-1}{j^{d}}} a_{1}^{\frac{1}{j^{d}}}
$$

so we must have $\lim _{j} a_{j}=0$, and this contradicts the hypothesis $\inf _{j} a_{j}=\lim _{j} a_{j}=$ $a>0$.

Acknowledgements. Laurent Moonens would like to acknowledge the support of the "Laboratory Ypatia of Mathematical Sciences", thanks to which the current collaboration has been possible. All authors are also grateful to the referees for their careful reading of the paper and their helpful suggestions. 


\section{References}

[1] Bateman, M.: Kakeya sets and directional maximal operators in the plane. - Duke Math. J. 147:1, 2009, 55-77.

[2] Berezhnoi, E. I., and A. V. Novikov: On the halo problem in the theory of differentiation of integrals. - Izv. Ross. Akad. Nauk Ser. Mat. 66:4, 2002, 3-26; English transl. in Izv. Math. $66: 4,2002,659-681$.

[3] Córdoba, A. and R. Fefferman: On differentiation of integrals. - Proc. Nat. Acad. Sci. U.S.A. 74:6, 1977, 2211-2213.

[4] D'Aniello, E., and L. Moonens: Averaging on $n$-dimensional rectangles. - Ann. Acad. Sci. Fenn. Math. 42:1, 2017, 119-133.

[5] De Guzmán, M.: Differentiation of integrals in $\mathbf{R}^{n}$. - Lecture Notes in Math. 481, SpringerVerlag, 1975.

[6] De Guzmán, M.: Real variable methods in Fourier analysis. - Mathematics Studies 46, NorthHolland, 1981.

[7] Hagelstein, P.: Maximal operators associated to sets of directions of Hausdorff and Minkowski dimension zero. - In: Recent advances in harmonic analysis and applications, Springer Proc. Math. Stat. 25, 2013, 131-138.

[8] Hare, K. E.: Maximal operators and Cantor sets. - Canad. Math. Bull. 43:3, 2000, 330-342.

[9] Katz, N. H.: A counterexample for maximal operators over a Cantor set of directions. - Math. Res. Lett. 3:4, 1996, 527-536.

[10] Krasnosel'skit, M. A., and Ya. B. Rutickit: Convex functions and Orlicz spaces. - Noordhoff, Groningen, 1961.

[11] Moonens, L.: Differentiating along rectangles, in lacunary directions. - New York J. Math. $22,2016,933-942$.

[12] Moonens, L., and J. Rosenblatt: Moving averages in the plane. - Illinois J. Math. 56:3, 2012, 759-793.

[13] Nagel, A., E. M. Stein, and S. Wainger: Differentiation in lacunary directions. - Proc. Nat. Acad. Sci. U.S.A. 75:3, 1978, 1060-1062.

[14] Rao, M. M., and Z. D. Ren: Theory of Orlicz spaces. - Marcel Dekker, New York, 1991.

[15] SJöGren, P., and P. SJöLIN: Littlewood-Paley decompositions and Fourier multipliers with singularities on certain sets. - Ann. Inst. Fourier (Grenoble) 31:1, 1981, 157-175.

[16] Stokolos, A. M.: On the differentiation of integrals of functions from Orlicz classes. - Studia Math. 94, 1989, 35-50.

[17] Stokolos, A. M.: Zygmund's program: some partial solutions. - Ann. Inst. Fourier (Grenoble) $55: 5,2005,1439-1453$.

[18] Stokolos, A. M., and P. Hagelstein: An extension of the Córdoba-Fefferman theorem on the equivalence between the boundedness of certain classes of maximal and multiplier operators. - C. R. Math. Acad. Sci. Paris 346:19-20, 2008, 1063-1065.

[19] Stokolos, A. M., and P. Hagelstein: Tauberian conditions for geometric maximal operators. - Trans. Amer. Math. Soc. 361:6, 2009, 3031-3040

[20] Strömberg, J.-O.: Weak estimates on maximal functions with rectangles in certain directions. - Ark. Mat. 15, 1977, 229-240. 